\title{
A branch-price-and-cut algorithm for the commodity constrained split delivery vehicle routing problem
}

\author{
Claudia Archetti, Nicola Bianchessi, M. Grazia Speranza \\ Department of Economics and Management, \\ University of Brescia, Brescia, Italy \\ Email: \{claudia.archetti,nicola.bianchessi,grazia.speranza\}@unibs.it
}

May 5, 2015

\begin{abstract}
We consider the Commodity constrained Split Delivery Vehicle Routing Problem (C-SDVRP), a routing problem where customers may request multiple commodities. The vehicles can deliver any set of commodities, and multiple visits to a customer are allowed only if the customer requests multiple commodities. If the customer is visited more than once, the different vehicles will deliver different sets of commodities. Allowing the splitting of the demand of a customer only for different commodities may be more costly than allowing also the splitting of each individual commodity, but at the same time it is easier to organize and more acceptable to customers. We model the C-SDVRP by means of a set partitioning formulation and present a branch-price-and-cut algorithm. In the pricing phase, the ng-path relaxation of a constrained elementary shortest path problem is solved with a label setting dynamic programming algorithm. Capacity cuts are added in order to strengthen the lower bound. We solve to optimality within 2 hours instances with up to 40 customers and 3 commodities per customer.
\end{abstract}

\section{Introduction}

The class of vehicle routing problems (VRPs) is one of the largest and most studied classes of combinatorial optimization problems. It is also one of the most computationally challenging classes. In VRPs capacitated vehicles are used to distribute 
products and satisfy the demand of a set of customers. With very few exceptions, vehicle routing problems model the distribution of a single product. The underlying assumption is that only the volume or the weight of products matters. Thus, even if several products are demanded by customers, the demand of a customer is expressed with a single number, the total weight or volume of the demanded products. Accordingly, the capacity of the vehicles is expressed in weight or volume, depending on which constraint is more binding. We refer to Toth and Vigo (2014) for a recent collection of chapters on different VRPs.

There exist distribution problems where different commodities, or groups of commodities, have to be treated individually. When different commodities require different temperatures, such as in the case of frozen, fresh and dry food, vehicles with compartments may be used. In waste management different kinds of waste cannot be mixed up and must be kept separated at any stage of the collection problem. If a single vehicle is dedicated to the collection, then the vehicle container must be divided in compartments, one for each kind of waste. We refer to Derigs et al. (2011) for a recent work on a multi-compartment routing problem, with fixed size of the compartments, and to references therein. Also for organizational purposes, different commodities may be handled by means of dedicated vehicles. Having an individual product loaded on a vehicle makes the loading and the unloading of a vehicle much simpler and avoids the need for any reshuffling of the load during the distribution process.

In this paper we consider the Commodity constrained Split Delivery Vehicle Routing Problem (C-SDVRP) introduced in Archetti et al. (2014), where different commodities are distributed to customers with capacitated vehicles. The vehicles are flexible and can deliver any set of commodities. A customer may be served by more than one vehicle but a single commodity can be delivered to each customer by one vehicle only. Each vehicle starts from a depot, visits a set of customers and returns to the depot at the end of the tour. Any customer may request any set of commodities. A vehicle that carries multiple commodities is totally flexible, that is it can carry any amount of any commodity, provided the constraint on the vehicle capacity is satisfied. We assume that the demand of a commodity of each customer does not exceed the capacity of a vehicle. The problem is a relaxation of the classical Capacitated Vehicle Routing Problem, as multiple visits to customers are allowed. On the other hand, it is more constrained than the Split Delivery Vehicle Routing Problem as the entire demand of a commodity must be delivered by the same vehicle. The concept is that, while allowing split deliveries may be unacceptable to customers, allowing different commodities to be delivered by different vehicles may be more acceptable. In addition to the applications mentioned above, the C-SDVRP models the situation where different companies share a warehouse and a fleet of vehicles for the distribution of their products. 
One commodity is associated with one company. A vehicle may deliver products of different companies to different customers or to the same customers. Different vehicles may serve the same customer only if they deliver products of different companies.

In Archetti et al. (2014) the C-SDVRP is introduced and compared with alternative ways of distributing multiple commodities, such as by allowing the splitting of individual commodities or by using vehicles dedicated to individual commodities. The tests were performed on 64 small instances, with 15 customers and up to 3 commodities, 80 mid-size instances with 20,40, 60, 80 customers and up to 3 commodities, and large instances with 100 customers. A branch-and-cut algorithm was able to solve 25 out of 64 small instances to optimality within 30 minutes. All the remaining instances were heuristically solved by creating multiple copies of each customer, one for each commodity required, and using a heuristic for the Capacitated Vehicle Routing Problem.

In this paper we focus on the exact solution of the C-SDVRP. We formulate the problem through a set partitioning formulation and adopt a branch-price-and-cut approach. In the pricing phase, the ng-path relaxation of a constrained elementary shortest path problem is solved by means of a label setting dynamic programming algorithm. Capacity cuts are added in order to strengthen the lower bound. We tested the algorithm on the small and mid-size instances tested in Archetti et al. (2014), with a time limit of 2 hours. We solved to optimality all small instances within a few seconds, except for three instances for which the computing time is larger than 100 seconds. For the mid-size instances, we could solve to optimality 19 out of 20 instances with 20 customers and 5 out of 20 instances with 40 customers. For all instances with up to 60 customers, except one, we found a lower and an upper bound. The average and maximum optimality gap are $0.63 \%$ and $2.41 \%$, respectively. Over all the mid-size instances, we improved 20 out of 80 best known solutions.

In Section 2 the C-SDVRP is described and in Section 3 the set partitioning formulation is presented. The structure of the branch-price-and-cut algorithm is described in Section 4, together with the formulation and solution of the pricing problem, and the branching scheme. The computational results are presented in Section 5 .

\section{Problem definition}

The Commodity constrained Split Delivery Vehicle Routing Problem (C-SDVRP) can be defined on a directed graph $G=(V, A)$ with vertex set $V=\{0, \ldots, n\}$ and arc set $A=\{(i, j): i \neq j ; i, j \in V\}$. The vertex set $V$ contains vertex 0 , 
representing the depot, and the set $N=\{1, \ldots, n\}$, representing the $n$ customers. A cost $c_{i j}$ is associated with each arc $(i, j) \in A$ and represents the non-negative cost of traversing arc $(i, j)$. Travel costs satisfy the triangle inequality. Let $K=$ $\{1, \ldots, m\}$ be the set of commodities that have to be distributed from the depot to the customers. The demand of commodity $k \in K$ to be delivered to customer $i \in N$ is denoted by $d_{i k}$. The set $K_{i}=\left\{k \in K \mid d_{i k}>0\right\}$ contains the commodities to be delivered to customer $i \in N$. We define as $F$ the fleet of identical vehicles that is available to serve the customers and as $Q$ the vehicle capacity. Vehicles are flexible and can deliver any subset of commodities. Each customer may be visited more than once. When a commodity is delivered by a vehicle to a customer, the entire amount requested by the customer is provided. Thus, multiple visits to a customer are allowed only if the customer requests multiple commodities. If a customer receives multiple visits, it means that the different vehicles will deliver different commodities. The objective is to find a set of routes serving all the customers in such a way that the total traveling cost is minimized.

\section{Formulation}

We model the problem by means of a set partitioning formulation making use of an exponential number of variables, each associated with a different feasible route. We adopt the following notation. We define as $R$ the set of all feasible routes. A route corresponds to a non empty cycle in graph $G$ starting from and ending at the depot. For each route $r \in R$, let $c^{r}=\sum_{(i, j) \in r} c_{i j}$ be the cost associated with the route. Then, let $a_{i k}^{r}, e_{i}^{r}$ and $b_{i j}^{r}$ be binary coefficients equal to 1 if commodity $k$ is delivered to customer $i \in N$, customer $i \in N$ is visited, and $\operatorname{arc}(i, j) \in A$ is traversed in route $r$, respectively, and 0 otherwise. The C-SDVRP can be formulated as follows: 


$$
\begin{aligned}
& \min \sum_{r \in R} c^{r} \lambda^{r} \\
& \text { s.t.: } \sum_{r \in R} a_{i k}^{r} \lambda^{r} \geq 1 \\
& \sum_{r \in R} \lambda^{r}=\phi \\
& \sum_{r \in R} e_{i}^{r} \lambda^{r}=z_{i} \\
& \sum_{r \in R} b_{i j}^{r} \lambda^{r}=x_{i j} \\
& 1 \leq z_{i} \leq \max \left\{\left|K_{i}\right|,|F|\right\} \text { and integer } \\
& i \in N, \quad k \in K_{i} \\
& \left\lceil\frac{\sum_{i \in N} \sum_{k \in K_{i}} d_{i k}}{Q}\right\rceil \leq \phi \leq|F| \text { and integer } \\
& 0 \leq x_{i j} \leq|F| \text { and integer } \\
& \lambda^{r} \in\{0,1\}
\end{aligned}
$$

where $x_{i j}$ and $z_{i}$ are integer variables representing the number of times the vehicles traverse $\operatorname{arc}(i, j) \in A$ and visit customer $i \in N$, respectively, $\phi$ is an integer variable representing the number of vehicles used, and $\lambda^{r}$ is a binary variable equal to 1 if route $r \in R$ is assigned to a vehicle. The objective function (1) aims at minimizing the total traveling cost. Constraints (2) ensure that all commodities requested by each customer will be delivered. Constraints (3) and (7) bound the number of vehicles that can be used. (4) and (6) bound the number of times a customer $i \in N$ can be visited. (5) and (8) bound the number of times an arc $(i, j) \in A$ can be traversed. Finally, (9) state that $\lambda^{r}$ are binary variables. Note that $x_{i j}$ can take any integer value between 0 and $|F|$ contrary to what happens in the Split Delivery Vehicle Routing Problem (SDVRP) where $x_{i j}$ is a binary variable for $i, j \in N$. In fact, in Archetti et al. (2014) the authors show that in the C-SDVRP arcs joining two customers can be traversed more than once.

\section{Branch-price-and-cut algorithm}

In the following we describe the algorithm designed to solve the set partitioning formulation (1)-(9) which will be referred to as the Master Problem (MP).

In order to solve the MP we design a branch-price-and-cut algorithm (Barnhart et al. (1998); Desaulniers et al. (2005)), that is a branch-and-bound algorithm 
where, at each node of the branch-and-bound tree, $\lambda^{r}$ variables are generated by means of column generation while addressing the linear relaxation of the problem associated with the node. We denote the linear relaxation of the MP, restricted to a subset of columns, as Restricted Linear Master Problem (RLMP). At each column generation iteration, a pricing problem is solved to generate negative reduced cost variables to be added to the RLMP. When no negative reduced cost variable is found it means that the Linear Master Problem (LMP), that is the linear relaxation of the MP, has been solved to optimality and the column generation algorithm ends. If the LMP solution is fractional, before applying branching rules, we possibly insert violated valid inequalities in the current RLMP and iterate its solution process in order to strengthen the bound.

In the following subsections the main components of the algorithm are described in details. The term path is used as a synonymous for route.

\subsection{Pricing problem formulation}

Given the solution values of the dual variables $\mu_{i k}, \rho, \theta_{i}, \sigma_{i j}$ associated with constraints (2), (3), (4), and (5) of the RLMP, respectively, the pricing problem can be modeled as follows:

$$
\begin{aligned}
& \min \sum_{(i, j) \in A} \bar{c}_{i j} X_{i j}-\sum_{i \in N} \sum_{k \in K_{i}} \mu_{i k} Y_{i k} \\
& \text { s.t.: } \sum_{j \in N} X_{0 j}=1 \\
& \sum_{(i, j) \in \delta^{+}(i)} X_{i j}=\sum_{(j, i) \in \delta^{-}(i)} X_{j i}=Z_{i} \\
& \sum_{(i, j) \in \delta^{+}(S)} X_{i j} \geq Z_{s} \\
& Y_{i k} \leq Z_{i} \\
& \sum_{k \in K_{i}} Y_{i k} \geq Z_{i} \\
& i \in N, k \in K_{i} \\
& i \in N \\
& \sum_{i \in N} \sum_{k \in K_{i}} d_{i k} Y_{i k} \leq Q \\
& S \subseteq N,|S| \geq 2, s \in S \\
& Y_{i k} \in\{0,1\} \\
& Z_{i} \in\{0,1\} \\
& i \in N, k \in K_{i} \\
& i \in N \\
& X_{i j} \in\{0,1\}
\end{aligned}
$$


where $\bar{c}_{i j}$ is the reduced cost of arc $(i, j) \in A\left(\bar{c}_{i j}=c_{i j}-\sigma_{i j}-\theta_{j}\right.$ if $j \neq 0$, $\bar{c}_{i j}=c_{i j}-\sigma_{i j}-\rho$ if $\left.j=0\right), \delta^{+}(S)=\{(i, j) \in A \mid i \in S, j \notin S\}$ and $\delta^{-}(S)=$ $\{(i, j) \in A \mid i \notin S, j \in S\}$. For the ease of notation, we write $\delta^{+}(i)$ and $\delta^{-}(i)$ when $S=\{i\}$. Binary variables $X_{i j}, Z_{i}$ and $Y_{i k}$ are equal to 1 if arc $(i, j) \in A$ is traversed, customer $i \in N$ is visited, and commodity $k \in K_{i}$ is delivered to customer $i \in N$, respectively, and 0 otherwise.

The objective function (10) aims at minimizing the reduced cost of the route. Constraints (11) and (12) are the degree constraints, whereas constraints (13) are subtour elimination constraints. Consistency between the $Y_{i k}$ and $Z_{i}$ variables is imposed through constraints (14) and (15). In particular, constraints (15) ensure that a delivery will take place at each customer visited. Finally, inequalities (16) impose the capacity constraint for the route.

The pricing problem combines an Elementary Shortest Path Problem with a 0-1 Knapsack Problem augmented by additional constraints. Actually, for a given path, determining the optimal values of the $Y_{i k}$ variables corresponds to solving the following problem:

$$
\begin{aligned}
& \max \sum_{i \in \bar{N}} \sum_{k \in K_{i}} \mu_{i k} Y_{i k} \\
& \text { s.t.: } \sum_{i \in \bar{N}} \sum_{k \in K_{i}} d_{i k} Y_{i k} \leq Q \\
& \sum_{k \in K_{i}} Y_{i k} \geq 1 \quad i \in \bar{N} \\
& Y_{i k} \in\{0,1\} \quad i \in \bar{N}, k \in K_{i}
\end{aligned}
$$

where $\bar{N}$ is the set of customers visited along the path. While addressing the SDVRP by means of column generation, the resulting pricing problem is such that in each solution path at most one customer will receive a split delivery greater than 1 and less than his demand (see Archetti et al. (2011)). Here, the structure of (20)-(23) is such that any customer visited along the solution path of (10)-(19) may receive any subset of the commodities requested.

In order to address the pricing problem (10)-(19), we formulate it as an Elementary Shortest Path Problem with Resource Constraints (ESPPRC) defined over an expanded graph $\mathcal{G}=(\mathcal{V}, \mathcal{A})$.

We define as $\mathcal{G}(i)=\left(\mathcal{V}_{i}, \mathcal{A}_{i}\right)$ the subgraph induced by the vertices corresponding to customer $i$. The set of vertices $\mathcal{V}_{i}$ is composed of vertices $v_{s}^{i}, v_{t}^{i}$ and $v_{k}^{i}$ for each $k \in K_{i}$. The arc set $\mathcal{A}_{i}$ includes the following arcs:

- $\operatorname{arcs}\left(v_{s}^{i}, v_{k}^{i}\right), k \in K_{i}$, with an associated cost equal to $-\mu_{i k}$; 
- $\operatorname{arcs}\left(v_{r}^{i}, v_{q}^{i}\right), r<q, r, q \in K_{i}$, with an associated cost equal to $-\mu_{i q}$;

- $\operatorname{arcs}\left(v_{k}^{i}, v_{t}^{i}\right), k \in K_{i}$, with an associated zero cost.

Moreover, a quantity equal to $d_{i k}$ is associated with each arc entering in $v_{k}^{i}, i \neq 0$ and $k \in K_{i}$, meaning that when vertex $v_{k}^{i}$ is visited, a quantity equal to $d_{i k}$ is delivered to $i$.

The vertex set $\mathcal{V}$ of the expanded graph includes two different vertices, $s$ and $t$, associated with the starting and ending depot, and the set $\mathcal{V}_{i}$ of $\left|K_{i}\right|+2$ vertices associated with each customer $i \in N$. The $\operatorname{arc} \operatorname{set} \mathcal{A}$ includes the sets of arcs $\mathcal{A}_{i}$, $i \in N$, and an $\operatorname{arc}\left(v_{t}^{i}, v_{s}^{j}\right)$ for each $\operatorname{arc}(i, j) \in A$, with cost equal to $\bar{c}_{i j}, v_{t}^{0}=s$ and $v_{s}^{0}=t$. Thus, each subgraph $\mathcal{G}(i)$ corresponds to an acyclic network (see Figure 1 ), where $v_{s}^{i}$ is the only vertex with incoming arcs originating from vertices not belonging to $\mathcal{V}_{i}$ and $v_{t}^{i}$ is the only vertex with outgoing arcs to vertices not belonging to $\mathcal{V}_{i}$.

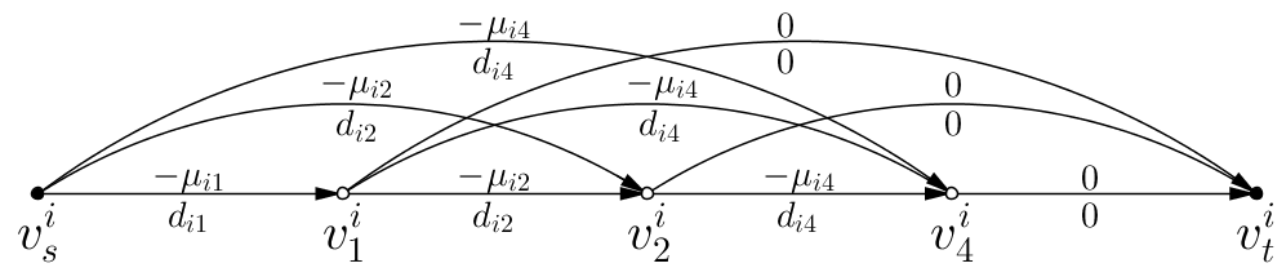

Figure 1: Acyclic network $\mathcal{G}(i)$ for customer $i \in N$, with $K_{i}=\{1,2,4\}$.

The pricing problem consists in finding the least (reduced) cost elementary path from $s$ to $t$ in $\mathcal{G}$, such that the quantity delivered along the path is less than or equal to $Q$. The ESPPRC defined over graph $\mathcal{G}$ is equivalent to (10)-(19).

The ESPPRC is NP-hard in the strong sense (see Dror (1994)). When the number of vertices is relatively large, the pricing problem may be very difficult to solve. We thus apply the ng-path relaxation of the problem that allows the generation of paths that may contain cycles (see Baldacci et al. (2011)).

\subsection{Pricing problem solution}

We solve the pricing problem by means of a label setting dynamic programming algorithm (see Irnich and Desaulniers (2005)). The main idea of this technique is to build feasible paths, starting from the source vertex of the graph, and extending them from vertex to vertex in all feasible directions. A label $(R, C, i)$ is associated with each partial path from the source of the graph to vertex $i$. Each component of the vector $R$ represents the consumption of a resource along the path until 
vertex $i$, whereas $C$ is the cost of the path. When $(R, C, i)$ is extended to another label $\left(R^{\prime}, C^{\prime}, j\right)$ associated with vertex $j$, the components of $R$ and the cost $C$ must be updated according to the resource consumption and the cost associated with arc $(i, j)$. Extensions must be feasible with respect to the consumptions of all the resources. To the sake of efficiency, dominance rules are applied to discard dominated paths reaching the same vertex. The solution is the path represented by the minimum cost label associated with vertex $t$.

Let us first consider the non-elementary version of the pricing problem. We solve it by using labels of the form $\left(q, C, v_{b}^{i}\right)$, where $b \in\{s, t\} \cup\left\{k \mid k \in K_{i}\right\}$ and $q$ is the quantity loaded on the vehicle when leaving vertex $v_{b}^{i}$. The initial label at vertex $v_{t}^{0}=s$ is $\left(0,0, v_{t}^{0}\right)$. At a given vertex $v_{b}^{i}$, a partial path associated with label $\left(q, C, v_{b}^{i}\right)$ is feasible if $q \leq Q$. Let us define $\tilde{c}_{i_{b} j_{b^{\prime}}}$ as the cost of $\operatorname{arc}\left(v_{b}^{i}, v_{b^{\prime}}^{j}\right) \in \mathcal{A}$. When arc $\left(v_{b}^{i}, v_{b^{\prime}}^{j}\right) \in \mathcal{A}$ is traversed, a label $\left(q, C, v_{b}^{i}\right)$ is extended to the label $\left(q^{\prime}, C^{\prime}, v_{b^{\prime}}^{j}\right)$, where $C^{\prime}=C+\bar{c}_{i_{b} j_{b^{\prime}}}$ and the extension rule for the $q$ component of the label is defined as follows. Along $\operatorname{arcs}\left(v_{t}^{i}, v_{s}^{j}\right)$ and $\left(v_{k}^{i}, v_{t}^{i}\right), i, j \in N, k \in K_{i}$, the resource consumption does not vary and its value is simply reported in the new label. Along $\operatorname{arcs}\left(v_{s}^{i}, v_{k}^{i}\right)$ and $\left(v_{r}^{i}, v_{q}^{i}\right), i \in N, k, r, q \in K_{i}$, the resource consumption is increased by $d_{i k}$ and $d_{i q}$, respectively. Dominance rules are then applied in order to discard paths that are dominated by other paths. Label $\left(q^{\prime}, C^{\prime}, v_{b}^{i}\right)$ dominates label $\left(q^{\prime \prime}, C^{\prime \prime}, v_{b}^{i}\right)$ if $q^{\prime} \leq q^{\prime \prime}, C^{\prime} \leq C^{\prime \prime}$, and one of the two conditions is strictly satisfied.

The ng-path relaxation of the pricing problem is obtained considering a further component in the label definition, that is a set $B$ of $|N|$ binary resources. A subset of customers $N_{i} \subseteq N$ is defined for each customer $i \in N$. $N_{i}$ includes $i$ and its closest $\nu$ neighbors, where $\nu$ is a positive integer value. Each element of $B$ is set to 0 in the initial label at vertex $s$. Let us consider then a partial path ending at vertex $v_{t}^{i} \in \mathcal{V}$. The extension of the path along $\operatorname{arc}\left(v_{t}^{i}, v_{s}^{j}\right) \in \mathcal{A}$ is feasible with respect to component $B$ if one of the following conditions holds:

- $v_{s}^{j}=t$, or equivalently $j=0$;

- $j \notin N_{i}$;

- $j \in N_{i}$ and $B(j)=0$.

When $\operatorname{arc}\left(v_{t}^{i}, v_{s}^{j}\right)$ is traversed, component $B$ is extended as follows. If $v_{s}^{j}=t, B^{\prime}(u)$ is set to 0 for each $u \in N$; otherwise $B^{\prime}(u)$ is set to 0 for all $u \notin N_{i} \cap N_{j}, B^{\prime}(u)$ becomes equal to $B(u)$ for all $u \in N_{i} \cap N_{j}$, and finally $B^{\prime}(j)$ is set to 1 . When one arc joining two vertices associated with the same customer is traversed, i.e., one of the $\operatorname{arcs}\left(v_{s}^{i}, v_{k}^{i}\right),\left(v_{k}^{i}, v_{q}^{i}\right)$ or $\left(v_{k}^{i}, v_{t}^{i}\right)$ with $k, r \in K_{i}$, component $B$ does not change.

Label $\left(q^{\prime}, B^{\prime}, C^{\prime}, v_{b}^{i}\right)$ dominates label $\left(q^{\prime \prime}, B^{\prime \prime}, C^{\prime \prime}, v_{b}^{i}\right)$ if $q^{\prime} \leq q^{\prime \prime}, C^{\prime} \leq C^{\prime \prime}$, $B^{\prime}(u) \leq B^{\prime \prime}(u)$ for each $u \in N_{i}$, and one of the conditions is strictly satisfied. 
An ng-path in graph $\mathcal{G}$ may contain a cycle $v_{s}^{i}=i_{1}, i_{2}, \ldots, i_{r}=v_{s}^{i}$, iff $i \notin N_{i_{q}}$ for some $q \in\{2, \ldots, r-1\}$ (see Baldacci et al. (2011) for more details). Note that, when we serve customer $i$, vertices $v_{s}^{i}$ and $v_{t}^{i}$ are visited together with at least one vertex $v_{k}^{i}$ with $k \in K_{i}$. Since graph $\mathcal{G}(i)$ is acyclic, a cycle in $\mathcal{G}$ is obtained when at least two customers are considered. Thus, a cycle $v_{s}^{i}=i_{1}, i_{2}, \ldots, i_{r}=v_{s}^{i}$ visits at least 7 vertices, i.e., $r \geq 7$ : three vertices correspond to the service of the first customers, 3 vertices correspond to the service of the second vertex and a further vertex is visited to return to the first customer.

Preprocessing. For a given set of dual variable values, each partial path reaching vertex $v_{s}^{i}, i \in N$, gives rise to at most $\beta_{i}$ non-dominated partial paths ending at node $v_{t}^{i}$, where $\beta_{i}$ is the number of Pareto-optimal solutions of the following bi-objective problem:

$$
\begin{gathered}
\max \sum_{k \in K_{i}} \mu_{i k} \mathcal{Y}_{k} \\
\min \sum_{k \in K_{i}} d_{i k} \mathcal{Y}_{k} \\
\text { s.t.: } \sum_{k \in K_{i}} d_{i k} \mathcal{Y}_{k} \leq Q \\
1 \leq \sum_{k \in K_{i}} \mathcal{Y}_{k} \leq\left|K_{i}\right| \\
\mathcal{Y}_{k} \in\{0,1\}
\end{gathered}
$$

where $\mathcal{Y}_{k}$ is a binary variable equal to 1 if commodity $k \in K_{i}$ is delivered to customer $i$, and 0 otherwise. The objective function (24) aims at maximizing the values of the dual variables $\mu_{i k}$ associated with commodities $k \in K_{i}$, while (25) aims at minimizing the capacity consumption. Constraints (26) and (27) bound the capacity consumption and the number of commodities that are delivered to customer $i$, respectively. The role of constraint (27) will be explained in Section 4.4 .

For each $i \in N$, we define $\mathcal{S}_{i}$, with $\left|\mathcal{S}_{i}\right|=\beta_{i}$, as the set of Pareto-optimal solutions of the corresponding problem (24)-(28). The Pareto-optimal solutions in set $\mathcal{S}_{i}$ can be represented by an acyclic network $\overline{\mathcal{G}}(i)$ with source and destination vertices $v_{s}^{i}$ and $v_{t}^{i}$, respectively, and a path between $v_{s}^{i}$ and $v_{t}^{i}$ for each solution in $\mathcal{S}_{i}$. Each path consists of a sequence of vertices $v_{k}^{i}, k \in K_{i}$, those corresponding to the commodities identified by the solution (see Figure 2). Set $\mathcal{S}_{i}$ is found by solving a SPPRC on the acyclic network $\mathcal{G}(i)$ (see Frieze (1976)) and keeping the non-dominated solutions. 


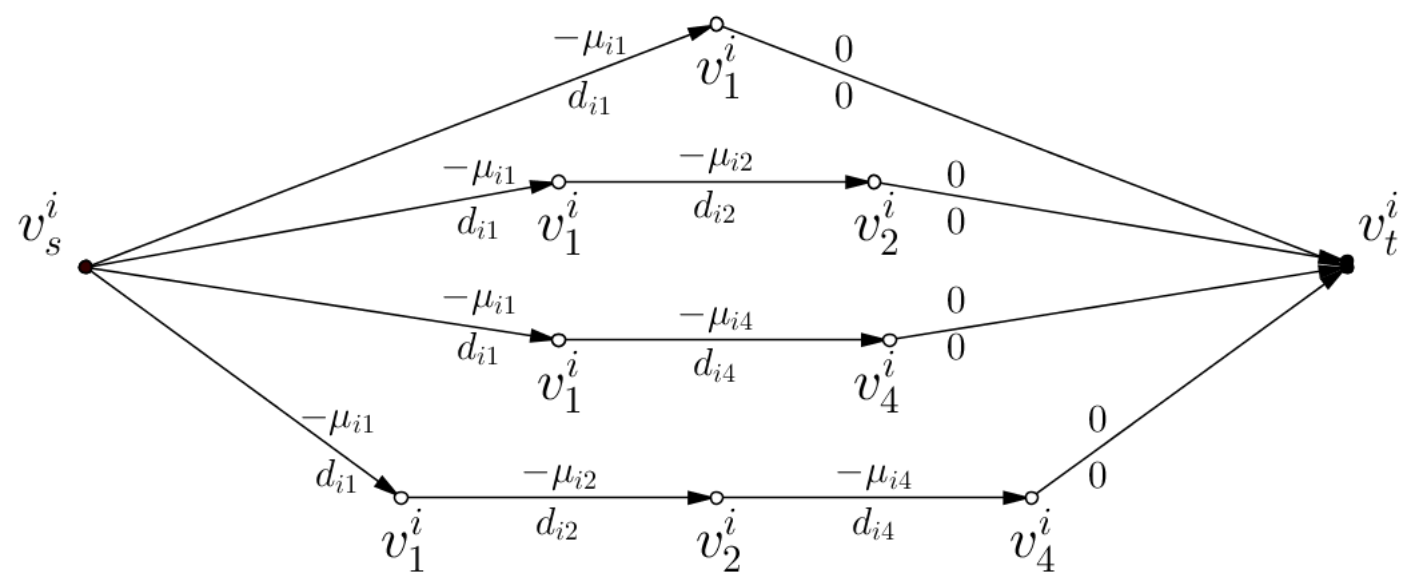

Figure 2: Acyclic network $\overline{\mathcal{G}}(i)$ for customer $i \in N$, with $K_{i}=\{1,2,4\}, d_{i 1}=2$, $d_{i 2}=3, d_{i 4}=5, \mu_{i 1}=4, \mu_{i 2}=2, \mu_{i 4}=5$, and $\mathcal{S}_{i}=\{\{1\},\{1,2\},\{1,4\},\{1,2,4\}\}$.

Replacing networks $\mathcal{G}(i)$ with networks $\overline{\mathcal{G}}(i)$ helps in avoiding redundant computations. The replacement is performed at every column generation iteration as the value of the dual variables changes at each iteration.

Acceleration strategies. In order to speed up the label setting algorithm we consider two different acceleration techniques. The first one is the decremental state space relaxation technique (Righini and Salani (2008)). The idea of this technique is to iteratively solve the pricing problem by enlarging, at each iteration, the set of vertices for which the elementarity is required. When combined with ng-paths, the procedure is such that sets $N_{i}$ are initially empty and are enlarged at each iteration. In particular, each customer $i \in N$ is associated with a set $M_{i}$ of binary values with $\left|M_{i}\right|=|N|$. At the first iteration, $M_{i}(i)=1$ and $M_{i}(u)=0$, $u \neq i, u \in N$. Then, the pricing problem is solved with a modified extension rule for component $B$ when $\operatorname{arc}\left(v_{t}^{i}, v_{s}^{j}\right) \in \mathcal{A}$ is traversed:

- if $v_{s}^{j}=t, B^{\prime}(u)=0, u \in N$;

- otherwise, $B^{\prime}(u)=0, u \notin \tilde{N}_{i} \cap \tilde{N}_{j} ; B^{\prime}(u)=B(u), u \in \tilde{N}_{i} \cap \tilde{N}_{j}$; and $B^{\prime}(j)=1$,

where $\tilde{N}_{i}=\left\{u \in N_{i} \mid M_{i}(u)=1\right\}$. Once vertex $v_{0}^{t}$ is reached and an $s-t$ path is constructed, the procedure checks whether this path is a feasible ng-path, i.e., it contains no cycle with respect to sets $N_{i}, i \in N$. If the path is a feasible ng-path, then the algorithm terminates. Otherwise, the first vertex $v_{s}^{u}$ in the path that defines a forbidden cycle is selected, $M_{i}(u)$ is set to 1 for each $i \in N$ such that $u \in N_{i}$, and the solution process is iterated. In practice, the elementarity is not 
required at the first iteration. The problem is solved and the first vertex $u$ which generates a cycle that is forbidden in the ng-path relaxation is added to all sets $\tilde{N}_{i}$ such that $u \in N_{i}$.

The second technique we implemented is the 2-cycle elimination proposed in Christofides et al. (1981). The predecessor of the last vertex visited is considered as a further component in the label definition. The predecessor is updated whenever $\operatorname{arc}\left(v_{t}^{i}, v_{s}^{j}\right) \in \mathcal{A}$ is traversed and remains unchanged when the path is extended through the other arcs of the expanded graph.

Heuristic column generation. To accelerate the solution of the LMP, at each column generation iteration heuristic versions of the label setting algorithm are applied before solving the pricing problem to optimality. While solving the pricing problem heuristically, the decremental state space relaxation technique is not applied and the dominance among labels is checked without considering the values of component $B$.

At each column generation iteration we derive from graph $\mathcal{G}$ a graph $\mathcal{G}^{\prime}$ where, for each $i \in N$, a subset of the outgoing $\operatorname{arcs}\left(v_{t}^{i}, v_{s}^{j}\right) \in \mathcal{A}, j \in N$, is considered. This subset includes the $\bar{n}_{a}$ outgoing arcs with lowest reduced cost values. Different heuristic algorithms are considered. The first two heuristics look for negative reduced cost paths in graphs $\mathcal{G}^{\prime}$ and $\mathcal{G}$, respectively, not allowing the possibility to perform split deliveries. The last two heuristics work again on graphs $\mathcal{G}^{\prime}$ and $\mathcal{G}$, respectively, but allow the possibility to perform split deliveries.

At each iteration of the column generation algorithm the heuristics are sequentially applied. The iteration finishes as soon as a heuristic succeeds in finding negative cost paths.

\subsection{Valid inequalities}

When the LMP solution is fractional and the node cannot be pruned, before starting the branching phase we look for violated capacity constraints which are formulated as follows:

$$
\sum_{r \in R} \sum_{(i, j) \in \delta^{+}(S)} b_{i j}^{r} \lambda^{r} \geq\left\lceil\frac{\sum_{i \in S} \sum_{k \in K_{i}} d_{i k}}{Q}\right\rceil S \subseteq N .
$$

In order to identify violated inequalities (29), we implemented the shrinking heuristic presented in Ralphs et al. (2003). In particular, two variants of the shrinking heuristic are implemented, namely the extended shrinking heuristic and the greedy shrinking heuristic. The reader is referred to Ralphs et al. (2003) for more details on these algorithms. 


\subsection{Branching}

When the optimal solution of the current $\operatorname{LMP}(\tilde{\boldsymbol{\lambda}}, \tilde{\phi}, \tilde{\mathbf{z}}, \tilde{\mathbf{x}})$ is fractional, different branching rules are hierarchically applied. The rules are presented in the following, in order of priority.

The first branching rule is on the fractional number of vehicles used. Then, the number of visits to each customer is considered. If $\tilde{z}_{i}$ is fractional for some $i \in N$, we branch on it. We give priority to $\tilde{z}_{i}$ lower than 2 . This allows us to forbid split deliveries to customer $i$ when $z_{i}$ is fixed to 1 . Moreover, when $z_{i}$ is constrained to be greater than or equal to $\alpha>1$, then the right-hand side term of constraint (27) is set equal to $\left|K_{i}\right|-(\alpha-1)$. For each class of priority, we choose the customer $i$ with fractional part of $\tilde{z}_{i}$ closest to 0.5 .

The third branching rule is related to the arc use, i.e., to variables $\tilde{\mathbf{x}}$. If $\tilde{x}_{i j}$ is fractional for some $(i, j) \in A$, then we branch on the use of arc $(i, j)$. As before, we choose the $\operatorname{arc}(i, j)$ with fractional part of $\tilde{x}_{i j}$ closest to 0.5 .

Finally, if none of the above branching decisions can be imposed, we consider each pair of different commodities $p$ and $q, p \in K_{i}, q \in K_{j}, i, j \in N$, and com-

pute $\kappa_{p q}=\sum_{r \in R} a_{p i}^{r} a_{q j}^{r} \tilde{\lambda}^{r}$, i.e. $\kappa_{p q}$ is the sum of the $\lambda$ variables associated with routes delivering commodities $p$ and $q$ to customers $i$ and $j$, respectively. If $\kappa_{p q}$ is fractional for some pair $p$ and $q$, we branch on it. On one branch we set $\kappa_{p q}=0$, meaning that commodities $p$ and $q$ must be delivered in different routes, while on the other branch we set $\kappa_{p q}=1$, meaning that commodities $p$ and $q$ must be delivered in the same route. The subproblem is modified accordingly. When $\kappa_{p q}$ is set to 0 , a new binary resource $B_{p q}$ must be introduced in the label definition in order to prevent the delivery of both commodities. $B_{p q}$ is set to 0 in the initial label and is increased by one whenever commodity $p$ or $q$ is delivered. Label $L^{\prime}$ is feasible w.r.t. resource $B_{p q}$ if $L^{\prime}\left(B_{p q}\right) \leq 1$, and may dominate label $L^{\prime \prime}$ if $L^{\prime}\left(B_{p q}\right) \leq L^{\prime \prime}\left(B_{p q}\right)$. When $\kappa_{p q}$ is set to 1 , two new binary resources, $B_{p}$ and $B_{q}$, are required. $B_{p}\left(B_{q}\right)$ is set to 0 in the initial label and is increased by one whenever commodity $p(q)$ is delivered. Label $L^{\prime}$ is feasible w.r.t. resource $B_{p}\left(B_{q}\right)$ if $L^{\prime}\left(B_{p}\right) \leq 1\left(L^{\prime}\left(B_{q}\right) \leq 1\right)$. In particular, label $L^{\prime}$ may dominate label $L^{\prime \prime}$ if $L^{\prime}\left(B_{p}\right)=L^{\prime \prime}\left(B_{p}\right)$ and $L^{\prime}\left(B_{q}\right)=L^{\prime \prime}\left(B_{q}\right)$, and is extended to vertex $t$ if $L^{\prime}\left(B_{p}\right)=L^{\prime}\left(B_{q}\right)$. We give priority to $\kappa_{p q}$ values where commodities $p$ and $q$ are associated with the same customer. For each class of priority, we choose the $\kappa_{p q}$ value closest to 0.5. This branching rule implements the Ryan and Foster (2003) rule and guarantees the correctness of the solution algorithm (see Barnhart et al. (1998)).

The search tree is explored according to a best-first-strategy. 


\section{$5 \quad$ Experimental results}

The branch-price-and-cut algorithm was implemented in $\mathrm{C}++$ and compiled in release mode under MS Visual Studio Express 2013 for Windows Desktop (64-bit version). The experiments were carried out on a 64-bit Windows machine, with Intel Xeon processor W3680, 3.33 GHz, and 12 GB of RAM. CPLEX 12.5 (64 bit version) was used to solve the linear relaxation of the MPs at each column generation iteration. The overall execution time limit for each run was set to 2 hours. A single thread was used in all experiments.

After a preliminary testing phase we fixed the parameters of the branch-priceand-cut algorithm as follows. The number of neighbors $\nu$ used while defining the ng-path relaxation of the pricing problem is set to $\left\lceil\frac{n}{2}\right\rceil$, provided that $10 \leq\left\lceil\frac{n}{2}\right\rceil \leq$ 15. If $\left\lceil\frac{n}{2}\right\rceil<10$ then $\nu=10$, while $\nu=15$ when $\left\lceil\frac{n}{2}\right\rceil>15$. The parameter $\bar{n}_{a}$ used in the heuristic column generation phase is set to $\min \left\{10,(n+1) * 2^{-4}\right\}$. Finally, violated inequalities are identified up to the fifth level of the branch-and-bound tree and, then, only when the branching has no impact on the lower bound, i.e., when the lower bound of the child node is equal to the lower bound of the father node.

\subsection{Test Instances}

We tested the branch-price-and-cut algorithm on benchmark instances for the CSDVRP proposed in Archetti et al. (2014). Three sets of instances with up to 3 commodities were generated: small instances with $n=15$, mid-size instances with $n=20,40,60,80$, and large instances with $n=100$. We consider only the small and the mid-size instances, as no significant result could be obtained on large instances.

Small instances have the following characteristics:

- Customer locations: The first 15 locations in the R101 and C101 Solomon's instances are considered;

- Number of commodities: Two and three;

- Probability that a customer requires a commodity: Two cases are considered. Probability set to $100 \%$ means that each customer requires each commodity. Probability set to $60 \%$ means that the probability that a customer requires a commodity is $60 \%$;

- Demand range: This data represents the interval for the generation of the demand of each customer for each commodity. Two intervals are considered: $[1 ; 100]$ and $[40 ; 60]$; 
- Vehicle capacity: Denoting as $d_{\max }=\max _{i \in C} \sum_{j=1}^{m} d_{i j}$, the vehicle capacity is generated as $Q=\alpha d_{\max }$. Four values of $\alpha$ are considered: 1.1, 1.5, 2 and 2.5 .

One instance for each of the 64 combinations of characteristics was generated.

Mid-size instances have a number of commodities equal to three, demand range $[1 ; 100]$, and $\alpha=1.5$. For each of the 16 combinations of value of $n$, type of instance (R101 and C101), and value of the probability (60\% and 100\%), 5 different instances were generated by randomly choosing customers from the original instance. The total number of mid-size instances is 80 .

\subsection{Computational results on small instances}

Detailed results on the set of small instances are reported in Table 1. The first four columns report data on the instance: name of the original instance, number of commodities $(m)$, probability that a customer requires a commodity $(p)$, demand range $(\Delta)$ and value of $\alpha$. The following $4+4$ columns refer to the computational results. The first four columns report statistics on the solution obtained at the root node of the branch-and-bound tree: the value of the LMP as a percentage of the final lower bound value $\left(\underline{z}_{L M P}^{*}\right)$, the time spent to compute $\underline{z}_{L M P}^{*}$ in seconds $\left(t_{L M P}\right)$, the value of the LMP once the capacity cuts are added as a percentage of the final lower bound value $\left(\underline{z}_{L M P+c u t s}^{*}\right)$ and the time spent to compute $\underline{z}_{L M P+\text { cuts }}^{*}$ $\left(t_{L M P+c u t s}\right)$. The last four columns report statistics on the final solution obtained by the branch-price-and-cut algorithm: the lower bound value $\left(\underline{z}^{*}\right)$, the upper bound value $\left(\bar{z}^{*}\right)$, the optimality percentage gap (gap \%) and the total execution time in seconds $(t)$.

The algorithm is able to solve to optimality all instances. In particular, it solves 41 instances out of the 64 in less than 1 second and takes more than 10 seconds for only 6 instances. As mentioned in the introduction, Archetti et al. (2014) proposed a branch-and-cut algorithm for the C-SDVRP which was able to solve 25 out of the 64 instances within a time limit of 30 minutes. 
Table 1: Small instances

\begin{tabular}{|c|c|c|c|c|c|c|c|c|c|c|c|c|}
\hline & \multicolumn{3}{|c|}{ Instance } & \multirow[b]{2}{*}{$\alpha$} & \multirow[b]{2}{*}{$\underline{z}_{L M P}^{*}$} & \multirow[b]{2}{*}{$\begin{array}{c}t_{L M P} \\
\text { (sec.) }\end{array}$} & \multirow{3}{*}{$\begin{array}{c}\text { root } \\
\underline{z}_{L M P+c u t s}^{*}\end{array}$} & \multirow[b]{2}{*}{$\begin{array}{c}t_{L M P+c u t s} \\
\quad(\mathrm{sec} .)\end{array}$} & \multirow[t]{2}{*}{$\underline{z}^{*}$} & \multirow[t]{2}{*}{$\bar{z}^{*}$} & \multirow{2}{*}{$\underset{(\%)}{\text { gap }}$} & $t$ \\
\hline & $m$ & $p$ & $\Delta$ & & & & & & & & & (sec.) \\
\hline C101 & 2 & 0.6 & {$[1 ; 100]$} & 1.1 & 100.00 & 0.168 & & - & 283.3404 & 283.3404 & 0 & 0.169 \\
\hline & & 0.6 & {$[40 ; 60]$} & 1.1 & 100.00 & 0.014 & - & - & 480.4342 & 480.4342 & 0 & 0.015 \\
\hline & & 1 & {$[1 ; 100]$} & 1.1 & 99.63 & 0.099 & 99.76 & 0.124 & 422.4965 & 422.4965 & 0 & 0.261 \\
\hline & & 1 & {$[40 ; 60]$} & 1.1 & 98.59 & 0.024 & 98.77 & 0.029 & 685.1662 & 685.1662 & 0 & 0.051 \\
\hline & & 0.6 & {$[1 ; 100]$} & 1.5 & 99.81 & 0.189 & 100.00 & 0.221 & 241.0386 & 241.0386 & 0 & 0.222 \\
\hline & & 0.6 & {$[40 ; 60]$} & 1.5 & 97.50 & 0.042 & 100.00 & 0.079 & 348.2731 & 348.2731 & 0 & 0.079 \\
\hline & & 1 & {$[1 ; 100]$} & 1.5 & 95.77 & 0.148 & 99.61 & 0.222 & 340.5474 & 340.5474 & 0 & 1.070 \\
\hline & & 1 & {$[40 ; 60]$} & 1.5 & 97.39 & 0.082 & 97.84 & 0.131 & 490.2973 & 490.2973 & 0 & 0.248 \\
\hline & & 0.6 & {$[1 ; 100]$} & 1.1 & 100.00 & 0.287 & - & - & 200.9092 & 200.9092 & 0 & 0.287 \\
\hline & & 0.6 & {$[40 ; 60]$} & 1.1 & 100.00 & 0.147 & - & - & 239.6829 & 239.6829 & 0 & 0.147 \\
\hline & & 1 & {$[1 ; 100]$} & 1.1 & 99.61 & 0.212 & 99.97 & 0.426 & 239.9424 & 239.9424 & 0 & 0.688 \\
\hline & & 1 & {$[40 ; 60]$} & 1.1 & 97.62 & 0.182 & 99.50 & 0.381 & 357.5929 & 357.5929 & 0 & 1.407 \\
\hline & & 0.6 & {$[1 ; 100]$} & 1.5 & 99.36 & 0.453 & 100.00 & 1.102 & 170.0453 & 170.0453 & 0 & 1.103 \\
\hline & & 0.6 & {$[40 ; 60]$} & 1.5 & 99.30 & 0.264 & 99.97 & 0.436 & 207.8259 & 207.8259 & 0 & 0.710 \\
\hline & & 1 & $1 ; 100]$ & 1.5 & 99.53 & 0.654 & 100.00 & 0.962 & 205.7830 & 205.7830 & 0 & 0.963 \\
\hline & & 1 & {$[40 ; 60]$} & 1.5 & 97.35 & 0.261 & 99.97 & 0.436 & 302.1813 & 302.1813 & 0 & 0.639 \\
\hline R101 & 2 & 0.6 & {$[1 ; 100]$} & 1.1 & 97.82 & 0.076 & 99.68 & 0.140 & 408.8971 & 408.8971 & 0 & 0.321 \\
\hline & & 0.6 & {$[40 ; 60]$} & 1.1 & 95.94 & 0.018 & 98.25 & 0.025 & 565.3383 & 565.3383 & 0 & 0.087 \\
\hline & & 1 & {$[1 ; 100]$} & 1.1 & 99.45 & 0.086 & 99.67 & 0.119 & 537.2029 & 537.2029 & 0 & 0.310 \\
\hline & & 1 & {$[40 ; 60]$} & 1.1 & 100.00 & 0.018 & - & - & 679.0232 & 679.0232 & 0 & 0.018 \\
\hline & & 0.6 & {$[1 ; 100]$} & 1.5 & 98.28 & 0.116 & 99.89 & 0.246 & 353.0119 & 353.0119 & 0 & 0.324 \\
\hline & & 0.6 & {$[40 ; 60]$} & 1.5 & 97.34 & 0.039 & 99.48 & 0.069 & 455.9724 & 455.9724 & 0 & 0.116 \\
\hline & & 1 & {$[1 ; 100]$} & 1.5 & 97.50 & 0.105 & 99.23 & 0.163 & 443.0829 & 443.0829 & 0 & 0.259 \\
\hline & & 1 & {$[40 ; 60]$} & 1.5 & 99.57 & 0.050 & 100.00 & 0.063 & 558.9565 & 558.9565 & 0 & 0.063 \\
\hline & & 0.6 & {$[1 ; 100]$} & 1.1 & 100.00 & 0.230 & - & - & 301.4316 & 301.4316 & 0 & 0.230 \\
\hline & & 0.6 & {$[40 ; 60]$} & 1.1 & 97.28 & 0.057 & 98.77 & 0.109 & 379.2848 & 379.2848 & 0 & 0.299 \\
\hline & & 1 & {$[1 ; 100]$} & 1.1 & 97.24 & 0.245 & 100.00 & 0.481 & 370.5561 & 370.5561 & 0 & 0.482 \\
\hline & & 1 & {$[40 ; 60]$} & 1.1 & 98.53 & 0.078 & 99.63 & 0.096 & 444.1868 & 444.1868 & 0 & 0.236 \\
\hline & & 0.6 & {$[1 ; 100]$} & 1.5 & 99.73 & 0.331 & 100.00 & 0.403 & 280.7646 & 280.7646 & 0 & 0.403 \\
\hline & & 0.6 & {$[40 ; 60]$} & 1.5 & 96.38 & 0.139 & 97.65 & 0.190 & 342.6854 & 342.6854 & 0 & 0.581 \\
\hline & & 1 & {$[1 ; 100]$} & 1.5 & 98.45 & 0.509 & 99.98 & 0.887 & 323.5761 & 323.5761 & 0 & 1.459 \\
\hline & & 1 & {$[40 ; 60]$} & 1.5 & 97.64 & 0.161 & 99.71 & 0.277 & 401.6087 & 401.6087 & 0 & 0.412 \\
\hline C101 & 3 & 0.6 & {$[1 ; 100]$} & 1.1 & 97.76 & 0.184 & 100.00 & 0.312 & 333.4709 & 333.4709 & 0 & 0.312 \\
\hline & & 0.6 & {$[40 ; 60]$} & 1.1 & 93.08 & 0.100 & 95.47 & 0.196 & 440.2241 & 440.2241 & 0 & 17.776 \\
\hline & & 1 & {$[1 ; 100]$} & 1.1 & 93.96 & 1.827 & 98.42 & 5.359 & 428.5164 & 428.5164 & 0 & 740.205 \\
\hline & & 1 & {$[40 ; 60]$} & 1.1 & 98.18 & 0.264 & 99.45 & 0.426 & 638.0919 & 638.0919 & 0 & 3.354 \\
\hline & & 0.6 & {$[1 ; 100]$} & 1.5 & 93.79 & 0.292 & 100.00 & 0.637 & 262.8069 & 262.8069 & 0 & 0.638 \\
\hline & & 0.6 & {$[40 ; 60]$} & 1.5 & 98.27 & 0.256 & 99.99 & 0.417 & 306.6363 & 306.6363 & 0 & 0.572 \\
\hline & & 1 & {$[1 ; 100]$} & 1.5 & 96.43 & 2.694 & 99.96 & 5.137 & 315.9600 & 315.9600 & 0 & 6.394 \\
\hline & & 1 & {$[40 ; 60]$} & 1.5 & 96.25 & 0.867 & 100.00 & 1.658 & 457.9430 & 457.9430 & 0 & 1.659 \\
\hline & & 0.6 & {$[1 ; 100]$} & 1.1 & 99.26 & 0.392 & - & - & 204.9380 & 204.9380 & 0 & 7.360 \\
\hline & & 0.6 & {$[40 ; 60]$} & 1.1 & 93.54 & 0.190 & 99.63 & 0.361 & 263.2896 & 263.2896 & 0 & 1.365 \\
\hline & & 1 & {$[1 ; 100]$} & 1.1 & 93.20 & 1.945 & 99.93 & 4.343 & 265.0623 & 265.0623 & 0 & 7.256 \\
\hline & & 1 & {$[40 ; 60]$} & 1.1 & 99.66 & 1.475 & 100.00 & 2.336 & 347.3580 & 347.3580 & 0 & 2.337 \\
\hline & & 0.6 & {$[1 ; 100]$} & 1.5 & 99.43 & 0.967 & 99.94 & 1.300 & 168.2958 & 168.2958 & 0 & 3.075 \\
\hline & & 0.6 & {$[40 ; 60]$} & 1.5 & 100.00 & 0.345 & - & - & 202.9044 & 202.9044 & 0 & 0.345 \\
\hline & & 1 & $1 ; 100]$ & 1.5 & 98.64 & 35.196 & 99.86 & 62.526 & 206.6970 & 206.6970 & 0 & 116.820 \\
\hline & & 1 & {$[40 ; 60]$} & 1.5 & 95.12 & 2.048 & 99.69 & 4.515 & 310.7978 & 310.7978 & 0 & 103.281 \\
\hline R101 & 3 & 0.6 & {$[1 ; 100]$} & 1.1 & 97.54 & 0.095 & 100.00 & 0.212 & 401.7502 & 401.7502 & 0 & 0.212 \\
\hline & & 0.6 & {$[40 ; 60]$} & 1.1 & 97.73 & 0.036 & 99.63 & 0.097 & 497.1385 & 497.1385 & 0 & 0.262 \\
\hline & & 1 & {$[1 ; 100]$} & 1.1 & 97.90 & 0.586 & 99.41 & 1.129 & 491.0411 & 491.0411 & 0 & 49.043 \\
\hline & & 1 & {$[40 ; 60]$} & 1.1 & 98.67 & 0.123 & 100.00 & 0.199 & 679.0232 & 679.0232 & 0 & 0.200 \\
\hline & & 0.6 & {$[1 ; 100]$} & 1.5 & 98.32 & 0.241 & 98.62 & 0.328 & 347.3693 & 347.3693 & 0 & 1.230 \\
\hline & & 0.6 & {$[40 ; 60]$} & 1.5 & 97.57 & 0.115 & 99.09 & 0.191 & 410.8130 & 410.8130 & 0 & 0.824 \\
\hline & & 1 & {$[1 ; 100]$} & 1.5 & 96.64 & 1.408 & 97.75 & 1.931 & 409.2905 & 409.2905 & 0 & 8.755 \\
\hline & & 1 & {$[40 ; 60]$} & 1.5 & 97.04 & 0.374 & 100.00 & 0.598 & 541.0336 & 541.0336 & 0 & 0.598 \\
\hline & & 0.6 & {$[1 ; 100]$} & 1.1 & 100.00 & 0.404 & - & - & 303.1439 & 303.1439 & 0 & 0.405 \\
\hline & & 0.6 & {$[40 ; 60]$} & 1.1 & 99.76 & 0.227 & 99.76 & 0.247 & 343.7159 & 343.7159 & 0 & 0.380 \\
\hline & & 1 & {$[1 ; 100]$} & 1.1 & 98.89 & 1.709 & 100.00 & 2.811 & 345.8351 & 345.8351 & 0 & 2.813 \\
\hline & & 1 & {$[40 ; 60]$} & 1.1 & 97.25 & 1.029 & 99.19 & 1.732 & 444.1868 & 444.1868 & 0 & 3.033 \\
\hline & & 0.6 & {$[1 ; 100]$} & 1.5 & 100.00 & 0.886 & - & - & 278.2234 & 278.2234 & 0 & 0.887 \\
\hline & & 0.6 & {$[40 ; 60]$} & 1.5 & 99.05 & 0.380 & 100.00 & 0.485 & 312.3100 & 312.3100 & 0 & 0.485 \\
\hline & & 1 & {$[1 ; 100]$} & 1.5 & 97.77 & 25.233 & 99.03 & 33.867 & 320.3490 & 320.3490 & 0 & 43.270 \\
\hline & & 1 & {$[40 ; 60]$} & 1.5 & 96.78 & 1.458 & 99.81 & 2.634 & 393.8357 & 393.8357 & 0 & 3.275 \\
\hline
\end{tabular}




\subsection{Computational results on mid-size instances}

Detailed results on the set of mid-size instances are reported in Table 2. The first four columns report statistics on the instance: name of the original instance, number of customers $(n)$, probability that a customer requires a commodity $(p)$, instance id $(i d)$. We recall that, in the set of mid-size instances, 5 instances are generated for each combination of characteristics and that the number of commodities is set to three, the demand range is $[1 ; 100]$ and $\alpha=1.5$. The following $4+4$ columns refer to the computational results and have the same meaning as in Table 1. The column 'nodes explored' gives the number of nodes explored in the branch-and-bound tree. Finally, the last two columns report the value of the best known feasible solution, taken from Archetti et al. (2014), and the percentage gap between this value and the lower bound reported in column $\underline{z}^{*}$, respectively.

The algorithm is able to solve to optimality all, except one, instances with 20 customers and 5 instances with 40 customers. The number of nodes explored is less than 100 for all instances except 4 with 20 customers. The low number of nodes explored for the instances with 60 and 80 customers is due to the fact that in many cases the computing time is entirely spent to compute the lower bound at the root node. 


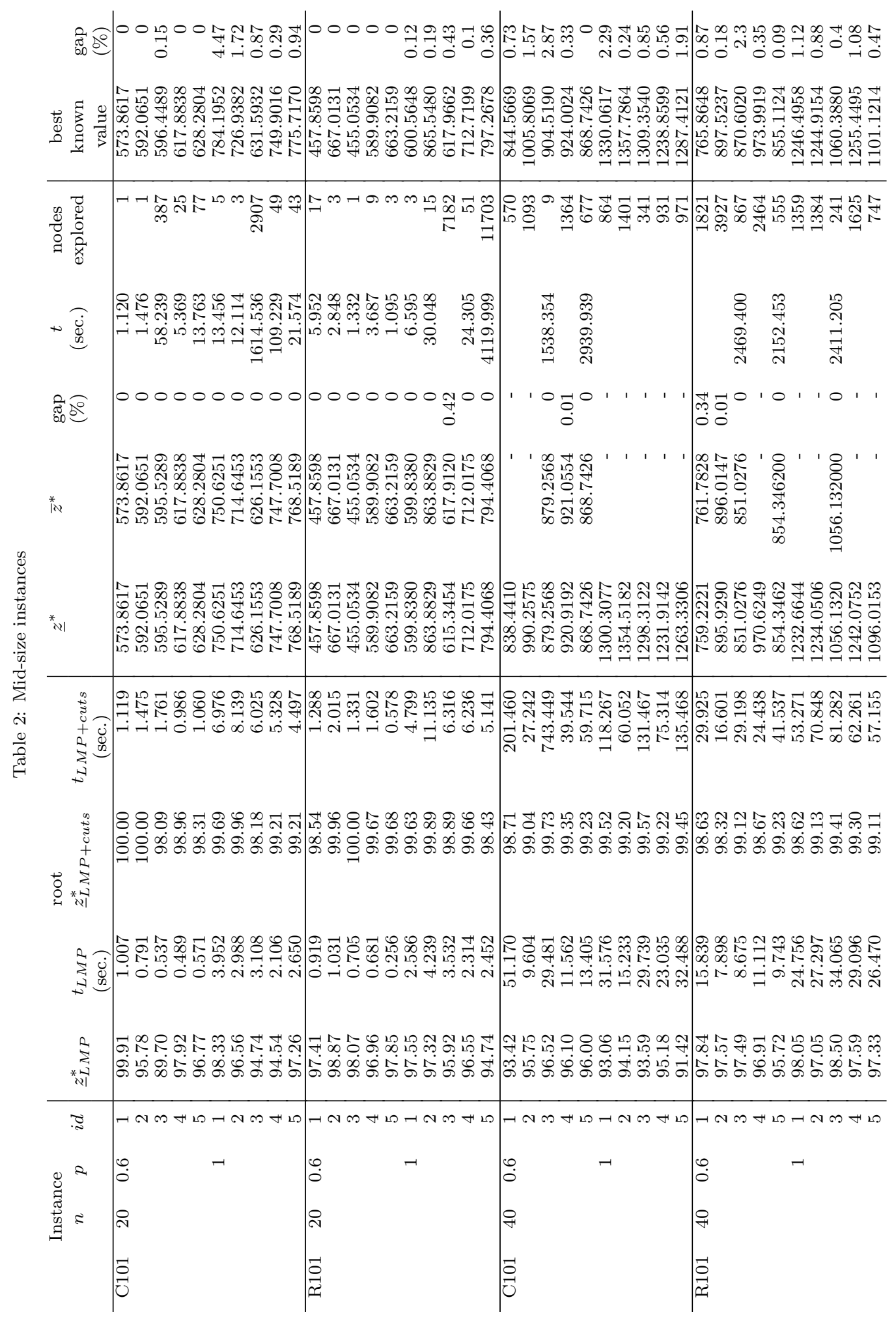




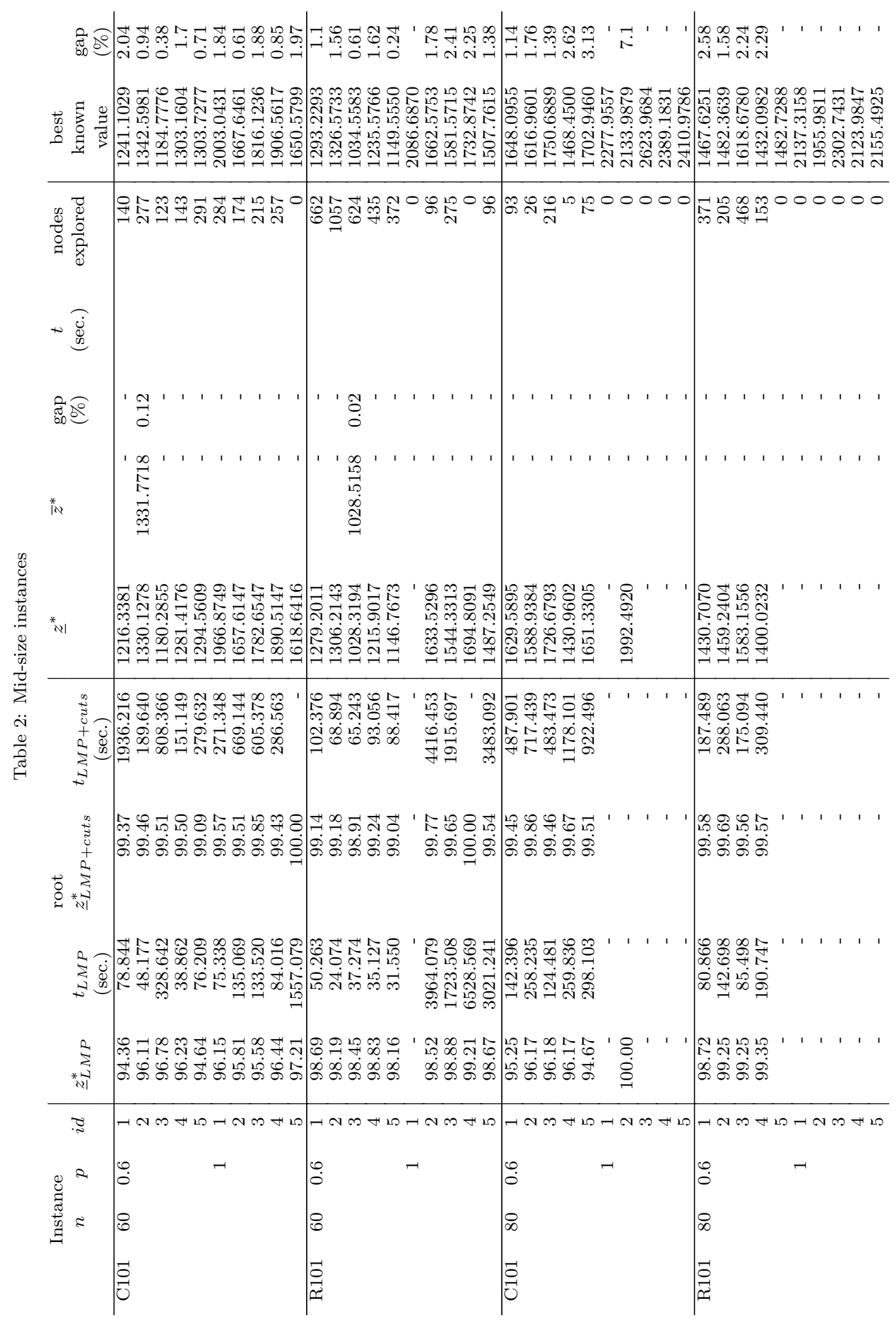


Table 3 reports a summary of the computational results on the mid-size instances. Instances are clustered on the basis of the values of $n$ and $p$. Each row corresponds to 10 instances. Column '\# opt.' reports the number of instances solved to optimality, '\# LB' is the number of times a lower bound was computed, 'av. gap' and 'max gap' report, in percentage, the average and maximum gap, respectively, between the computed lower bound and the value of the best known solution (the best between the solution computed by the branch-price-and-cut algorithm and the one reported in Archetti et al. (2014)), and '\# best known' is the number of times the branch-price-and-cut found or improved the best feasible solution. In parentheses we report the number of improved feasible solutions with respect to the best known reported in Archetti et al. (2014).

As expected, instances of larger size are more difficult to solve. Moreover, when $p=1$ the difficulty of solving the problem increases. This is due to the fact that, when $p=1$, all customers require all the commodities while a smaller number of commodities are required when $p=0.6$. In the latter case, the expanded graph used for the solution of the pricing problem is smaller. Out of the 30 instances for which the algorithm finds a solution, this solution is better than the best known solution found in Archetti et al. (2014) on 20 cases. For all instances, except one, with up to 60 customers a lower bound is found and the maximum optimality gap is $2.41 \%$.

Table 3: Summary of results on mid-size instances

\begin{tabular}{cc|rrccr}
$n$ & & & & av. & max & \# best \\
\# opt. & \# LB & gap & gap & known \\
\hline 20 & 0.6 & 10 & 10 & 0.00 & 0.00 & $10(1)$ \\
& 1 & 9 & 10 & 0.04 & 0.42 & $10(10)$ \\
\hline 40 & 0.6 & 4 & 10 & 0.30 & 1.57 & $7(6)$ \\
& 1 & 1 & 10 & 0.94 & 2.29 & $1(1)$ \\
\hline 60 & 0.6 & 0 & 10 & 0.95 & 2.04 & $2(2)$ \\
& 1 & 0 & 9 & 1.66 & 2.41 & 0 \\
\hline 80 & 0.6 & 0 & 9 & 2.08 & 3.13 & 0 \\
& 1 & 0 & 1 & 7.10 & 7.10 & 0
\end{tabular}

Finally, in Table 4 we report some information concerning the solution of the mid-size instances for which the branch-price-and-cut algorithm was able to compute a feasible solution. These are the 30 instances for which an upper bound $\left(\bar{z}^{*}\right)$ is provided in Table 2. The first 4 columns have the same meaning as in Table 2. We then report the number of routes performed and the number of customers served in each route. When the number of customers visited in a route is underlined, the route contains at least one split customer. In the following column we report the number of split deliveries performed in each route that visits at least one 
split customer. Finally, the last two columns report the total number of customers that are served by two and three routes, respectively. In all except one instance at least one customer receives a split delivery while in only 4 instances we have customers which are served by 3 routes. Moreover, in 20 cases over 30 the number of routes containing at least one split customer is greater than or equal to half of the total number of routes. The number of split customers in a single route is in most cases 1 or 2 but is as large as 5 in one case. 


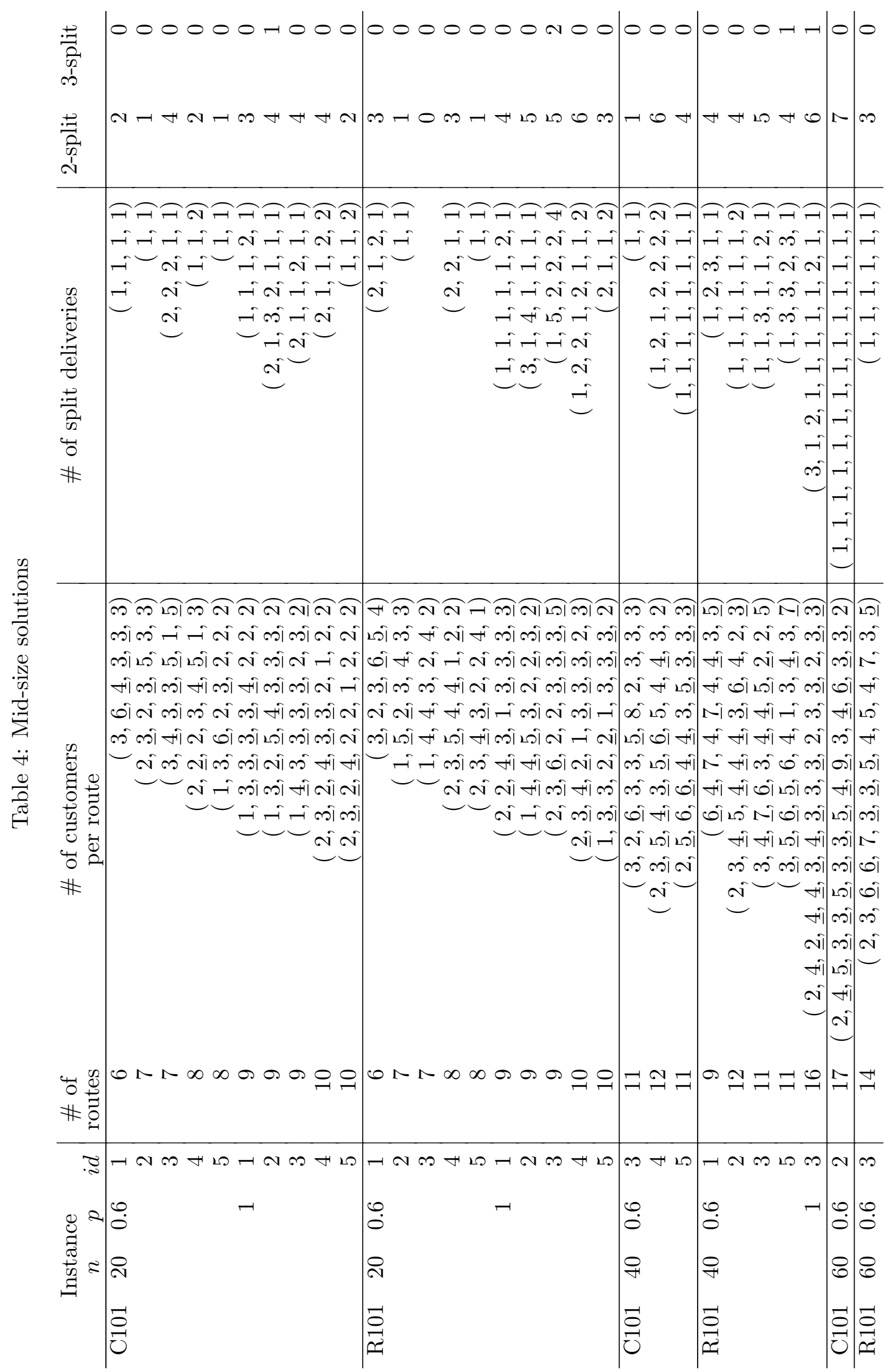




\section{Conclusions}

We presented in this paper a branch-price-and-cut algorithm for the solution of a routing problem that explicitly considers the presence of multiple commodities in a distribution problem and allows multiple visits to a customer as long as a commodity is not split between vehicles. The algorithm solves instances with up to 40 customers and 3 commodities, more than doubling the size 15 of the instances previously solved to optimality. In particular, if we consider the 60 medium size instances with at most 60 customers, the algorithm is able to compute or improve the best known upper bound values in $50 \%$ of cases (30/60), and only in $5 / 59$ cases the lower bound computed is far from the best known upper bound by more than $2 \%$. The algorithm was not able to compute the lower bound for one instance, but for the remaining 59/60 instances the average and maximum optimality gaps are $0.63 \%$ and $2.41 \%$, respectively. Also, for the instances with at most 60 customers we improved 20 of the best known solutions.

Future research should be devoted to the development of a heuristic for the solution of the C-SDVRP. In fact, the problem was solved heuristically by Archetti et al. (2014) by transforming it into a VRP while no ad-hoc heuristic has been proposed. Several variants of the C-SDVRP that consider multiple commodities would also deserve to be studied.

\section{References}

C. Archetti, N. Bianchessi, and M. G. Speranza. A column generation approach for the split delivery vehicle routing problem. Networks, 58:241-254, 2011.

C. Archetti, A. M. Campbell, and M.G. Speranza. Multi-commodity vs. singlecommodity routing. Transportation Science, 2014. doi: 10.1287/trsc.2014.0528.

R. Baldacci, A. Mingozzi, and R. Roberti. New route relaxation and pricing strategies for the vehicle routing problem. Operations Research, 59:1269-1283, 2011.

C. Barnhart, E. L. Johnson, G. L. Nemhauser, M. W. P. Savelsbergh, and P. H. Vance. Branch-and-price: column generation for solving huge integer programs. Operations Research, 46:316-329, 1998.

N. Christofides, A. Mingozzi, and P. Toth. Exact algorithms for the vehicle routing problem, based on spanning tree and shortest path relaxations. Mathematical Programming, 20:255-282, 1981. 
U. Derigs, J. Gottlieb, J. Kalkoff, M. Piesche, F. Rothlauf, and U. Vogel. Vehicle routing with compartments: applications, modelling and heuristics. OR Spectrum, 33:885-914, 2011.

G. Desaulniers, J. Desrosiers, and M. Solomon, editors. Column generation. Springer, 2005.

M. Dror. Note on the complexity of the shortest path models for column generation in VRPTW. Operations Research, 42:977-978, 1994.

A.M. Frieze. Shortest path algorithms for knapsack type problems. Mathematical Programming, 11:150-157, 1976.

S. Irnich and G. Desaulniers. Shortest path problems with resource constraints. In G. Desaulniers, J. Desrosiers, and M.M. Solomon, editors, Column Generation, pages 33-65. Springer, 2005.

T.K. Ralphs, L. Kopman, W.R. Pulleyblank, and L.E. Trotter. On the capacitated vehicle routing problem. Mathematical Programmming, 94:343-359, 2003.

G. Righini and M. Salani. New dynamic programming algorithms for the resource constrained shortest path problem. Networks, 51:155-209, 2008.

D.M. Ryan and B.A. Foster. On the capacitated vehicle routing problem. Mathematical Programmming, 94:343-359, 2003.

P. Toth and D. Vigo, editors. Vehicle Routing: Problems, Methods and Applications. Series on Optimization. MOS-SIAM, Philadephia, Second edition, 2014. 